\title{
Successful 2-Stage Pacemaker Implantation for Atrioventricular Block in a Low- Birth-Weight Infant with Congenital Heart Disease: Case Report
}

\author{
Leigh A. Kline, ${ }^{1}$ Matthew A. Hazle, ${ }^{2}$ Yoshio Ootaki ${ }^{1}$ \\ ${ }^{1}$ Division of Pediatric Cardiothoracic Surgery, Wake Forest Baptist Health, Winston-Salem, NC \\ ${ }^{2}$ Pediatric Cardiology, Wake Forest Baptist Health, Winston-Salem, NC
}

\section{ABSTRACT}

Congenital heart block is a potentially life-threatening condition with high morbidity and mortality, especially in the presence of congenital heart disease. We present the case of a low-body-weight premature infant with complex single ventricle congenital heart disease and high-grade atrioventricular block. A 2-staged pacing approach provided atrio-ventricular synchrony and allowed her to grow until a permanent dualchamber pacemaker system could be implanted.

\section{CASE REPORT}

A female infant was born at 29 weeks' gestation, weighing 1,840 $\mathrm{g}$ with Apgar scores 5 and 7 at 1 and 5 minutes, respectively. For maternal reasons, delivery was performed at an outside hospital via urgent cesarean section. The fetus was known to have complex congenital heart disease, congenital atrioventricular block, fetal bradycardia, and hydrops fetalis. The infant was bradycardic after birth, with heart rates in the 50- to 60-bpm range and clinical evidence of poor perfusion. The infant was intubated and started on an epinephrine drip before transport to our hospital.

Postnatal echocardiogram confirmed complex congenital heart disease with unbalanced complete atrioventricular septal defect (left ventricular hypoplasia), double-outlet right ventricle, pulmonary valve stenosis, and interrupted inferior vena cava with azygos connection (heterotaxy syndrome). Initial electrocardiogram showed second-degree heart block with $2: 1$ conduction, resulting in a ventricular rate of 50 to 60 bpm (Figure 1).

Because of the presence of single-ventricle congenital heart disease, it was determined that dual-chamber pacing would be needed for optimal hemodynamics. Given the infant's small size, temporary epicardial pacing wires were placed on the right atrium and the right ventricle via a median sternotomy on day of life 0 . Although she subsequently required medical treatment for necrotizing enterocolitis, she remained hemodynamically stable and was able to

Received October 14, 2020; accepted November 24, 2020.

Correspondence: Yoshio Ootaki, Medical Center Boulevard, Winston-Salem, NC 27157; 336-716-2218 (e-mail: yotaki@wakehealth.edu). gain weight with nasogastric tube feeding without any other major complications.

At 7 weeks of age and a body weight of 2,640 g, urgent pacemaker replacement was performed owing to increased ventricular lead threshold and intermittent noncapture. Epicardial bipolar pacing leads were placed on the surface of the left atrium and left ventricle via a left anterolateral thoracotomy. The pacemaker generator (AdaptaTM model ADDRS1; Medtronic, Minneapolis, $\mathrm{MN}$ ) was implanted underneath the fascia of the left rectus abdominal muscle (Figure 2).

The postoperative course was uneventful, with good hemodynamics. Follow-up echocardiograms showed qualitatively normal systemic right ventricular systolic function and mild common atrioventricular valve insufficiency. The family then moved out of state, and she was transferred to another hospital for further management and cardiac catheterization, followed by bidirectional Glenn surgery.

\section{DISCUSSION}

Congenital heart block is a rare condition, with an overall incidence of $\sim 1$ in 22,000 births. Although presentation can vary, the majority of patients are identified prenatally or immediately after birth owing to the presence of bradycardia. Infants with both congenital heart block and congenital heart disease are at high risk for poor outcomes, and these patients often require pacemaker implantation in the neonatal period [Friedman 2003]. In the setting of congenital heart disease, pacemaker implantation is often indicated for neonates and

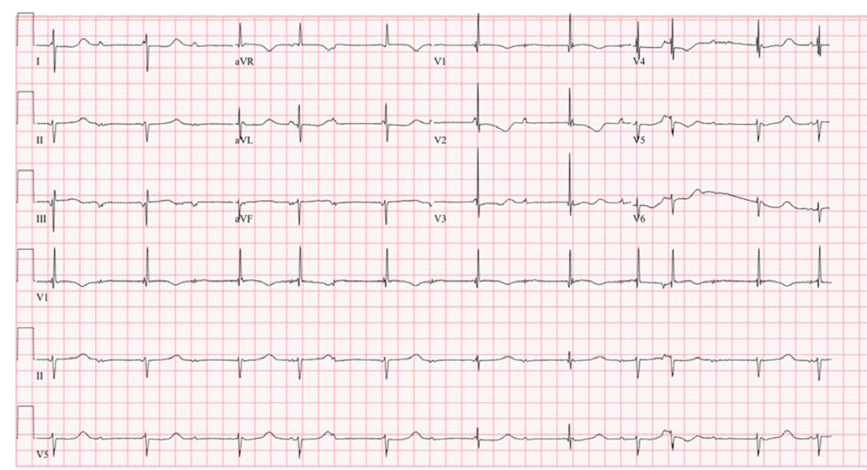

Figure 1. Original preoperative electrocardiogram at birth. Note the high-grade atrioventricular block heart rate, $60 \mathrm{bpm}$. 


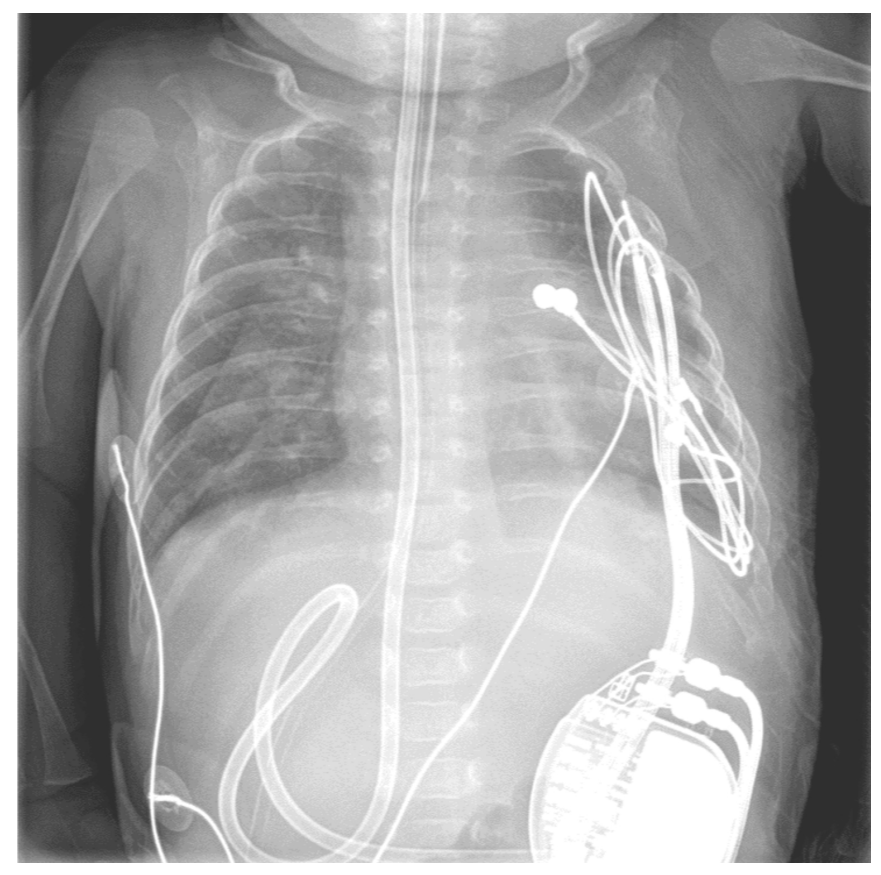

Figure 2. Chest-abdominal x-ray after placement of dual-chamber pacing system.

infants when the ventricular rate is $<70 \mathrm{bpm}$ [Villain 2008]. In this case, maintaining atrioventricular synchrony via dualchamber pacing was thought to be necessary because of the presence of single-ventricle congenital heart disease [Heinemann 2003, Connolly 1996].

In a study of permanent dual-chamber epicardial pacing in neonates with congenital heart block, 3 of the 4 patients with heterotaxy syndrome died after the procedure, whereas no patients with structurally normal hearts died [Kelle 2007]. Another study of neonates paced with temporary ventricular pacing systems showed that 5 of 6 patients with structurally normal hearts lived, whereas 6 of 7 patients with congenital heart disease died before discharge [Glatz 2008]. The study hypothesized that although dual-chamber pacing may be advantageous for establishing hemodynamic stability, it results in a poorly tolerated abrupt increase in ventricular rate. This is a consideration in a newborn with poor ventricular systolic function. In the case presented here, however, ventricular function was adequate after birth, and it was felt that dual-chamber pacing was critical to establish atrioventricular synchrony owing to single-ventricle physiology.

This case was especially challenging because of the presence of prematurity and low body weight. Infants $<3 \mathrm{~kg}$ have been shown to have a significantly greater risk for surgical complications such as infection and wound dehiscence after permanent epicardial pacemaker implantation [Chaouki 2017]. The use of a temporary system allowed time for somatic growth, potentially decreasing the surgical risk of dual-chamber permanent pacemaker placement. Considerations for a 2-staged approach include need for 2 surgical procedures and 2 incision sites, in addition to increased hospital length of stay. Although the 2-staged approach shows promise as a safer option for pacing of high-risk infants, there are a number of additional risks associated with the approach that would need to be further evaluated and their significance further studied.

In summary, this case demonstrates successful implantation of a permanent dual-chamber epicardial pacemaker system via a 2 -staged approach in a low-birth-weight premature infant with complex congenital heart disease.

\section{REFERENCES}

Chaouki AS, Spar DS, Khoury PR, et al. Risk factors for complications in the implantation of epicardial pacemakers in neonates and infants. Heart Rhythm 2017;14:206-210.

Connolly SJ, Kerr C, Gent M, Yusuf S. Dual-chamber versus ventricular pacing. Circulation 1996;94:578-583.

Friedman DM, Duncanson LJ, Glickstein J, Buyon JP. A review of congenital heart block. Images Paediatr Cardiol 2003;5:36-48.

Glatz AC, Gaynor JW, Rhodes LA, et al. Outcome of high-risk neonates with congenital complete heart block paced in the first 24 hours after birth. J Thorac Cardiovasc Surg 2008;136:767-773.

Heinemann MK, Gass M, Breuer J, Ziemer G. DDD pacemaker implantation after Fontan-type operations. Pacing Clin Electrophysiol 2003;26:492-495.

Kelle AM, Backer CL, Tsao S, et al. Dual-chamber epicardial pacing in neonates with congenital heart block. J Thorac Cardiovasc Surg 2007;134:1188-1192.

Villain E. Indications for pacing in patients with congenital heart disease. Pacing Clin Electrophysiol 2008;31:S17-S20. 